\title{
Intracranial relapse as a solitary mass of ocular adnexal lymphoma of the mucosa-associated lymphoid tissue type
}

Jung-Ho Kim ${ }^{1}$, Young-Woo Jeon², Byung-Ock Choi ${ }^{3}$, Gyeongsin Park ${ }^{4}$, Yong-Kil Hong ${ }^{5}$, Suk-Woo Yang ${ }^{6}$, and Seok-Goo Cho ${ }^{2}$

${ }^{1}$ Division of Hematology and Oncology, Department of Internal Medicine, The Armed Forces Capital Hospital, Seongnam; ${ }^{2}$ Department of Hematology, Catholic Blood and Marrow Transplantation Center, Departments of ${ }^{3}$ Radiation Oncology, ${ }^{4}$ Pathology, ${ }^{5}$ Neurosurgery, and ${ }^{6}$ Ophthalmology, College of Medicine, Seoul St. Mary's Hospital, The Catholic University of Korea, Seoul, Korea
Received: May 18, 2015

Revised : August 31, 2015 Accepted: September 13, 2015

\section{Correspondence to}

Seok-Goo Cho, M.D.

Department of Hematology, Catholic Blood and Marrow Transplantation Center, College of Medicine, Seoul St. Mary's Hospital, The Catholic University of Korea, 222 Banpo-daero, Seocho-gu, Seoul 06591, Korea Tel: +82-2-2258-6052

Fax: +82-2-599-3589

E-mail: chosg@catholic.ac.kr
To the Editor,

Ocular adnexal lymphoma (OAL) is a rare disease, constituting $1 \%$ to $2 \%$ of non-Hodgkin lymphomas and $8 \%$ of all extranodal lymphomas [1]. Among OALs, a heterogeneous group of malignancies, extranodal marginal zone lymphoma (EMZL) of the mucosa-associated lymphoid tissue (MALT) type is the most common, accounting for up to $80 \%$ of primary OALs [1]. Despite the close proximity of the ocular region to the central nervous system (CNS), ocular adnexal MALT lymphoma (OAML) rarely shows intracranial involvement [2]. There have been no previous reports of intracranial re- lapse as a solitary cerebral mass of OAML after successful treatment.

A 64-year-old woman attended the Catholic Blood and Marrow Transplantation Center (CBMTC) in February 2010 and was evaluated by one of the authors for a chief complaint of unilateral ocular hyperemia for 10 months. The patient did not have a headache, painful eyeball movements, or any other visual symptoms. Physical examination of the right eye showed an irregular reddish patch involving the inferolateral aspect of the conjunctiva (Fig. 1A). Findings of left eye examination were unremarkable. Magnetic resonance imaging
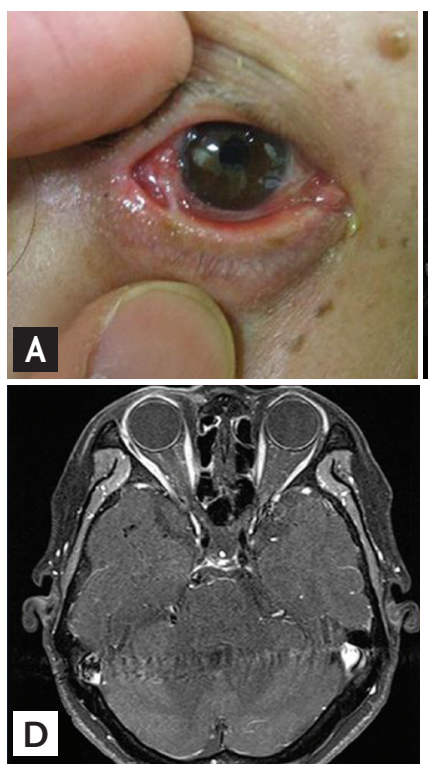
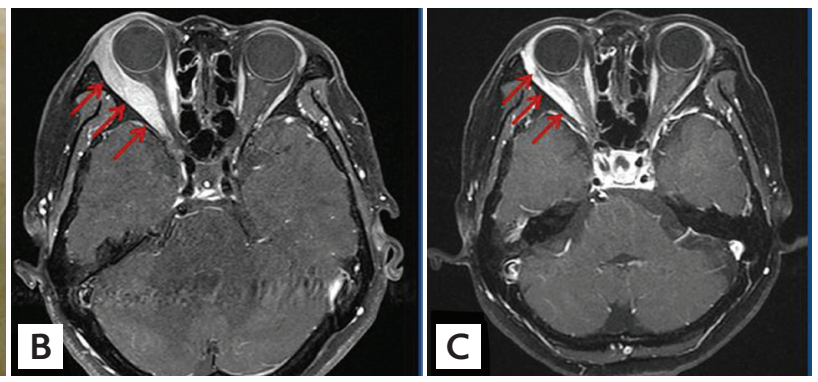

Figure 1. (A) Irregular reddish patch on the inferolateral aspect of conjunctiva. (B) In magnetic resonance imaging (MRI) at the time of diagnosis, T1 weighted image showed $1.1 \times 5.2 \times 3.2 \mathrm{~cm}$ sized mass (arrows) with of low signal intensity involving right lateral rectus muscle and lacrimal gland. (C) Follow-up MRI showed a decreased thickness of primary mass (arrows) after the third cycle of rituximab, cyclophosphamide, vincristine, and prednisolone treatment. (D) MRI showed further improvement without evidence of residual tumor after the intensity-modulated radiation therapy. 
(MRI) revealed a $1.1 \times 5.2 \times 3.2 \mathrm{~cm}$ sized mass involving the right lacrimal gland and right lateral rectus muscle, extending to the superior portion of the inferior rectus muscle through the intraconal and extraconal areas (Fig. 1B). An incisional biopsy was performed immediately to determine the final diagnosis. The specimen taken from the right orbital mass showed diffuse atypical lymphoid infiltration consisting of small lymphocytes with irregular nuclei (Fig. 2A). Immunohistochemistry showed atypical lymphocytes that were strongly positive for CD2O (Fig. 2B), and Ki67 index was $10 \%$. The pathologists concluded that the histologic findings are consistent with marginal zone B-cell lymphoma. Cerebrospinal fluid examination was not performed. No involvement of EMZL was detected on bone marrow examination or imaging studies, including positron emission tomography. The initial disease stage was IE (single extranodal) based on the Ann Arbor staging system.

The patient initially received a regimen of rituximab, cyclophosphamide, vincristine, and prednisolone (R-CVP) every 3 weeks. After three cycles of the immunochemotherapy, treatment response was assessed by MRI, which showed a decreased size of primary tumor in the right lateral rectus muscle (Fig.
1C). The patient had grade- 4 neutropenia during the systemic treatment, and deteriorated with complications such as nausea, vomiting, and general weakness. As she seemed to be intolerable to further administrations of systemic chemoimmunotherapy, intensity-modulated radiation therapy with 30 Gy was applied with curative intent. At the time of response evaluation after treatment, no evidence of residual tumor was observed on MRI (Fig. 1D). Although the patient underwent an operation for a right eye cataract which was a complication of radiation therapy diagnosed 2 years after the regional therapy, she lived a healthy life for 4 years. During that period, there was no evidence of recurrent disease on annually performed MRI.

The patient was admitted to the CBMTC again in September 2014 with a chief complaint of disorientation. She suffered from headache, nausea, and vomiting. An emergent brain MRI revealed a $3.8 \times 2.8 \mathrm{~cm}$ sized solitary mass with surrounding hemorrhage and prominent edema in the left basal ganglia (Fig. $3 \mathrm{~A}$ and $\left.{ }_{3} \mathrm{~B}\right)$. We found no evidence of local recurrence of OAML in brain MRI and additional ocular MRI was not performed. Since the acute symptoms of the patients seemed to be caused by increased intracranial pressure, we attempted to relieve symptoms with
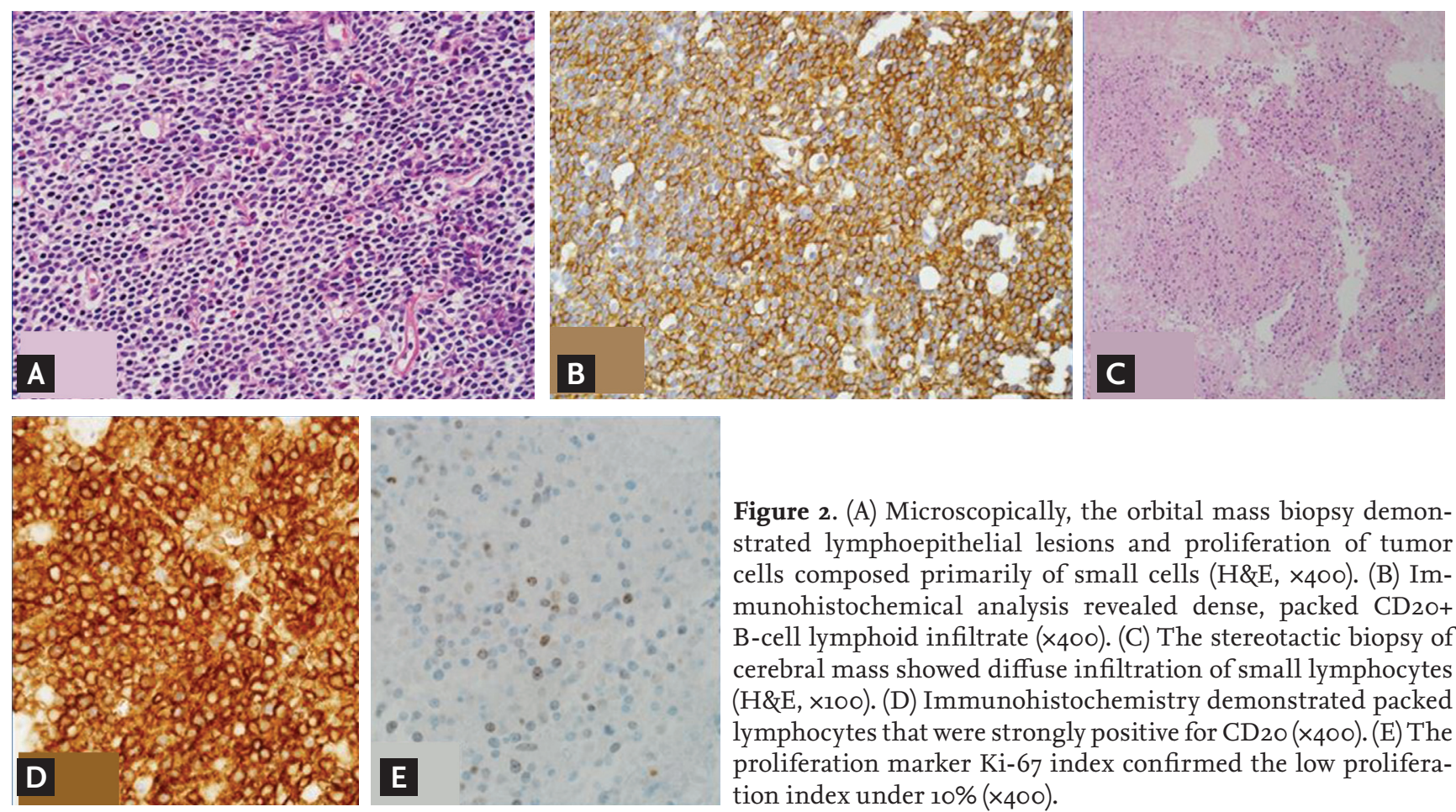

Figure 2. (A) Microscopically, the orbital mass biopsy demonstrated lymphoepithelial lesions and proliferation of tumor cells composed primarily of small cells (H\&E, $\times 400)$. (B) Immunohistochemical analysis revealed dense, packed $\mathrm{CD} 2 \mathrm{O}+$ B-cell lymphoid infiltrate $(\times 400)$. (C) The stereotactic biopsy of cerebral mass showed diffuse infiltration of small lymphocytes (H\&E, $\times 100)$. (D) Immunohistochemistry demonstrated packed lymphocytes that were strongly positive for $\mathrm{CD} 2 \mathrm{O}(\times 400)$. (E) The proliferation marker Ki-67 index confirmed the low proliferation index under 10\% (×400). 

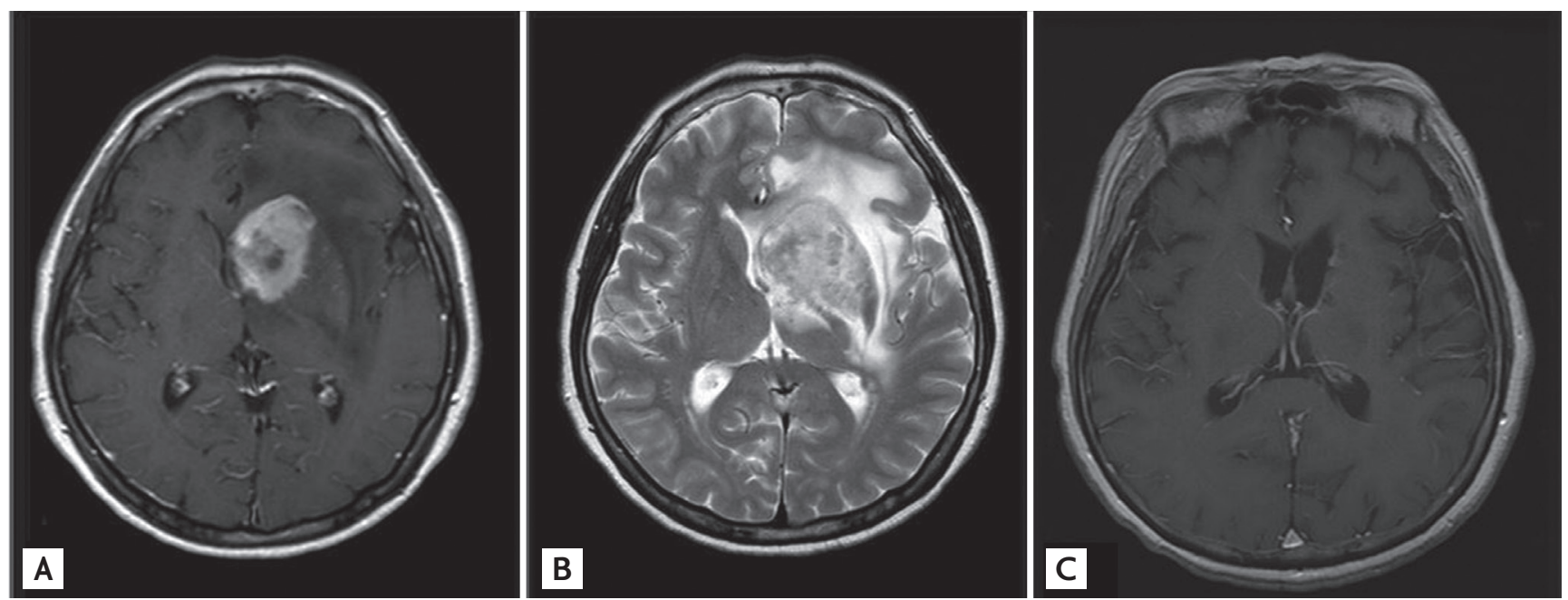

Figure 3. (A) In the emergent magnetic resonance imaging, T1 weighted image showed a $38 \times 28$ mm sized heterogenously and strongly enhancing mass in left basal ganglia, mainly involving the head of caudate nucleus. (B) T2 weighted image showing enhancing mass with surrounding hemorrhage and edema, arousing the midline shift of cerebrum. (C) After radiotherapy and systemic chemotherapy, a radiologic complete remission was achieved.

intravenous administrations of dexamethasone and mannitol. Stereotactic biopsy of the cerebral mass was scheduled in consultation with neurosurgeons. After reviewing the obtained specimens, pathologists concluded that the histological diagnosis was relapsed EMZL, presenting an identical pattern to the specimen of primary OAML on both immunohistochemistry and histopathology (Fig. $2 \mathrm{C}-2 \mathrm{E}$ ). Whole brain radiotherapy using 40 Gy dramatically reduced the size of the recurrent tumor and mass-related symptoms. Systemic chemotherapy was subsequently performed and it achieved complete remission (Fig. $3 \mathrm{C}$ ) after the fifth cycle of a high-dose methotrexate $\left(3.5 \mathrm{~g} / \mathrm{m}^{2}\right)$ regimen.

OALs were described at first in 1952. They are the most frequent malignant tumors of ocular adnexa. In the Florida Cancer Registry, they represent up to $55 \%$ of all orbital tumors [1]. Between 1975 and 2001, the incidence of OALs increased $6.3 \%$ annually [1]. The majority of OALs are primary extranodal neoplasms, while $10 \%$ to $32 \%$ are secondary tumors in patients with disseminated lymphomas [1]. According to the World Health Organization, the most common subtype among OALs is EMZL of the MALT type, accounting for $35 \%$ to $80 \%$ of cases, followed by follicular lymphoma, diffuse large B-cell lymphoma (DLBCL), and other less common types of lymphoma [1]. Pre- vious studies have shown that the relative incidence of OAML varies among different populations. The incidence of OAML among primary OALs is higher in Asian countries, such as Korea (86\% to $98 \%$ ), than in Western countries (50\% to $78 \%$ ) [3].

OAMLs usually present as localized disease at the time of diagnosis. Most OAMLs have been recorded as stage IE tumors based on the Ann Arbor staging system. Only $5 \%$ of newly diagnosed OAML patients have nodal involvement, and $8 \%$ have bone marrow infiltration [1]. With a disproportionate staging distribution, this classical staging system, first used in 1971, is thought to be weak in classifying the prognostic group of OALs. The American Joint Committee on Cancer subsequently suggested a new staging system for OALs based on the tumor, node, metastasis (TNM) classification. Our patient was classified as having T2dNoMo disease based on the TNM system and stage IE, the earliest stage in the Ann Arbor staging system, even though she experienced a disastrous relapse in the CNS. Our study in 2011 showed that the TNM staging system seems to overcome the weaknesses in the Ann Arbor staging system and allows better stratification of OAML according to prognosis [3]. In the study, 92.6\% of cases (50/54) were classified as Ann Arbor stage IE. Compared to TiNoMo cases $(n=26)$, cases staged above TiNoMo $(n=28)$ had a significantly 
higher rate of disease progression [3].

OAMLs are primarily low-grade lymphomas and highly responsive to primary therapy, including radiotherapy and chemotherapy. Many previous studies have documented favorable clinical outcomes for OAML [1]. Although previous reports have shown variability in the relapse rate of OAML after radiotherapy, recurrence in distant sites seems to be uncommon. A 1,998 case report demonstrated recurrent EMZL arising from the left psoas muscle, 18 months after initial treatment of OAML with radiotherapy [4]. However, distant relapse of OAML generally occurs in other organs of MALT, such as the contralateral orbit, breast, and parotid gland [2].

There are few reports of OAML involving the CNS $[1,3]$. A review article introduced a case of MALT lymphoma from the lateral and inferior rectus muscles, involving the cavernous sinus and dura of the anterior cranial fossa [1]. Moreover, the case was diagnosed with lymphomatous meningitis, based on a positive result on cerebrospinal fluid analysis [1]. Another article presented two cases of OAML, either initially or upon relapse, with diffuse involvement of the optic nerve and extension to the dura mater and cavernous sinus [3]. All of the cases with CNS involvement previously reported seemed to have primary tumors infiltrating into CNS directly. But as far as we know, none of previous study demonstrated a patient of OAML with CNS involvement as a solitary cerebral mass. This case is the first report that shows a pathologically proven relapse of OAML as a solitary cerebral mass. But there is a limitation that we could not exclude a chance of pathologic transformation to high grade lymphoma, because the small sections of tissue obtained from a stereotactic biopsy were unable to define a histology of the whole cerebral lesion. As the majority of cases of lymphoma with CNS involvement have aggressive histologic types such as DLBCL, clinicians should mind the possibility of pathologic transformation when they detect a CNS involvement during a clinical course of low grade lymphoma.
In 1998, Restrepo et al. [5] showed that CNS prophylaxis with radiotherapy or chemotherapy for OAL is unnecessary. None of their 71 enrolled patients had CNS relapse in the 2 years of follow-up [5]. As both CNS relapse and CNS involvement at diagnosis are rare, the current trend in treatment protocols to exclude CNS prophylaxis appears to be reasonable. Here, we present a case with intracranial relapse as a solitary mass of OAML 4 years after successful initial treatment. Despite the rarity of CNS involvement, we suggest that patients with OAML should be evaluated adequately when there are suspicious symptoms or signs of CNS manifestation.

Keywords: Lymphoma, B-cell, marginal zone; Intracranial; Recurrence

\section{Conflict of interest}

No potential conflict of interest relevant to this article was reported.

\section{REFERENCES}

1. Stefanovic A, Lossos IS. Extranodal marginal zone lymphoma of the ocular adnexa. Blood 2009;114:501-510.

2. Lee SE, Paik JS, Cho WK, et al. Feasibility of the TNMbased staging system of ocular adnexal extranodal marginal zone lymphoma of mucosa-associated lymphoid tissue (MALT lymphoma). Am J Hematol 2011;86:262266.

3. Bayraktar S, Stefanovic A, Montague N, Davis J, Murray $T$, Lossos IS. Central nervous system manifestations of marginal zone B-cell lymphoma. Ann Hematol 2010;89:1003-1009.

4. Cahill MT, Moriarty PA, Kennedy SM. Conjunctival 'MALToma' with systemic recurrence. Arch Ophthalmol 1998;116:97-99.

5. Restrepo A, Raez LE, Byrne GE Jr, et al. Is central nervous system prophylaxis necessary in ocular adnexal lymphoma? Crit Rev Oncog 1998;9:269-273. 